\title{
Enhanced recovery after surgery (ERAS) might be a standard care in radical prostatectomy: a systematic review and meta-analysis
}

\author{
Ziqi Ye ${ }^{1 \#}$, Jie Chen ${ }^{2 \#}$, Taoye Shen ${ }^{1}$, Hongyu Yang ${ }^{1}, J_{i e}$ Qin $^{3}$, Feiyue Zheng ${ }^{4}$, Yuefeng Rao ${ }^{1}$ \\ ${ }^{1}$ Department of Pharmacology, the First Affiliated Hospital, College of Medicine, Zhejiang University, Hangzhou 310003, China; ${ }^{2}$ Department of \\ Pharmacy, the Second Affiliated Hospital, College of Medicine, Zhejiang University, Hangzhou 310009, China; ${ }^{3}$ Department of Urology, the First \\ Affiliated Hospital, College of Medicine, Zhejiang University, Hangzhou 310003, China; ${ }^{4}$ Department of Pharmacy, Sir Run Run Shao Hospital, \\ College of Medicine, Zhejiang University, Hangzhou 310016, China. \\ Contributions: (I) Conception and design: Z Ye, J Chen; (II) Administrative support: Y Rao; (III) Provision of study materials or patients: Z Ye, J Qin, \\ F Zheng; (IV) Collection and assembly of data: Z Ye, J Chen, T Shen; (V) Data analysis and interpretation: Z Ye, J Chen, H Yang; (VI) Manuscript \\ writing: All authors; (VII) Final approval of manuscript: All authors. \\ "These authors contributed equally to this work. \\ Correspondence to: Yuefeng Rao. Department of Pharmacology, the First Affiliated Hospital, College of Medicine, Zhejiang University, 79 Qingchun \\ Road, Hangzhou 310003, China. Email: raoyf@zju.edu.cn.
}

Background: Several enhanced recovery after surgery (ERAS) protocols for radical prostatectomy (RP) have been reported in recent years. Nonetheless, there is no sufficient evidence to support the implementation of ERAS as a standard of care modality.

Methods: A search was done in the PubMed, Embase, Clinical Trials.gov, Cochrane Library, CNKI Library databases and reference lists to identify relevant studies from inception until May 2019 to be included in the study. A systematic review of five randomized controlled trials (RCTs), one prospective cohort study and four retrospective studies covering 3,803 patients, comparing ERAS with conventional care was performed. Outcomes of interest for the study were intraoperative outcomes (operation time and blood loss), postoperative outcomes (hospital stay, catheter stay, first defecation and first anal exhaust) and postoperative complications. Random events meta-analyses were performed. Sensitivity analysis was also performed to determine whether the results of the meta-analysis were robust.

Results: Notably, ERAS group had significantly shorter hospital stay [overall standardized mean difference $(\mathrm{SMD})=-1.65,95 \%$ confidence interval $(\mathrm{CI}):-2.53,-0.76, \mathrm{P}<0.001]$, shorter time to first defecation (overall $\mathrm{SMD}=-1.56,95 \% \mathrm{CI}:-2.71,-0.42, \mathrm{P}=0.008$ ), shorter time to first anal exhaust (overall SMD $=-1.23$, $95 \% \mathrm{CI}:-1.97,-0.50, \mathrm{P}=0.001)$ and lower incidence of nausea [overall risk ratio $(\mathrm{RR})=0.62,95 \% \mathrm{CI}: 0.40$, $0.94, \mathrm{P}=0.024]$ compared to the conventional group. There was no statistical difference in intraoperative outcomes, catheter stay and other postoperative complications between the two groups $(\mathrm{P}>0.05)$.

Conclusions: The data presented so far consistently show that ERAS may be utilized as a standard of care in RP treatment.

Keywords: Enhanced recovery after surgery (ERAS); conventional care; radical prostatectomy (RP); hospital stay; postoperative complications; systematic review

Submitted Aug 27, 2019. Accepted for publication Feb 12, 2020.

doi: 10.21037/apm.2020.04.03

View this article at: http://dx.doi.org/10.21037/apm.2020.04.03 


\section{Introduction}

Prostate cancer (Pca) is the most common genitourinary tract tumor in men (1). Radical prostatectomy (RP) is a first-line treatment for low and intermediate-risk localized Pca patients (2). RP can be either open or minimally invasive. Currently, minimally invasive RPs are widely applied. These are laparoscopic radical prostatectomy (LRP) and robot-assisted laparoscopic radical prostatectomy (RALP). Particularly, RALP has become the mainstay invasive treatment for localized Pca. Compared to open RP, minimally invasive RP is an effective and widely accepted treatment for localized Pca (3-5). Moreover, it reduces perioperative outcomes such as blood loss (6). However, open RP is still an option in some countries.

Enhanced recovery after surgery (ERAS) is a protocol aimed to reduce perioperative complications as well as the physical and psychological stress of surgical trauma. It is also described as fast-track surgery (FTS), because it accelerates patient rehabilitation, shortens the hospitalization period and reduce the medical costs. It was first performed by Kehlet in 1997 (7). It includes a series of evidence-based procedures, such as surgical, nursing, medical, anesthetic and perioperative managements (8).

A high number of Pca patients are elderly people. As such, comorbidities are very common thus necessitating the reduction of perioperative complications and acceleration of patient's recovery. ERAS has been widely applied in patients undergoing colorectal, breast and gastrointestinal surgery (9-12). However, its application in patients undergoing urological surgery is relatively low (13). To date, there is no meta-analysis to compare the efficacy and safety of ERAS to conventional care in patients undergoing RP. With more data available currently, a systematic review and metaanalysis was performed to assess whether ERAS should be considered as a standard care for patients undergoing RP.

\section{Methods}

\section{Search strategy}

We performed a comprehensive literature search on Embase, PubMed, the Clinicaltrials.gov (http://clinicaltrials. gov/), the Cochrane Library and CNKI Library to identify clinical trials that compared ERAS and conventional care. The search was done in all data published before May 2019. The search terms included: "ERAS", "Enhanced recovery after surgery", "Enhanced recovery", "perioperative management", "Fast-track surgery", "FTS", "Radical prostatectomy" and "Prostate cancer". We also screened the reference lists of review articles. Additional studies were also retrieved by manual search in relevant journals. We exclusively included studies which were published in English and Chinese.

\section{Inclusion and exclusion criteria}

Studies were selected according to the Preferred Reporting Items for Systematic Reviews and Meta-Analyses (PRISMA) statement (14). Clinical trials that met the four listed criteria were included:

(I) Randomized phase II, III, and IV trials;

(II) Patients who underwent RP;

(III) Participants who had received ERAS compared to conventional care and

(IV) Trails with available events, event rates and sample sizes to enable determination of efficacy and safety of ERAS.

Trials were excluded if:

(I) They involved animal research;

(II) They were reviews only;

(III) Had abstracts only;

(IV) Had overlapping data and

(V) Those studies without standard mean difference (SMD), risk ratio (RR), odds ratio (OR), hazard ratio (HR) and $95 \%$ confidence intervals (CIs).

\section{Data extraction and quality assessment}

Two reviewers independently did literature screening, data extraction, and quality assessment of the trials. A third reviewer was involved to have a consensus were the two reviewers disagreed. From each article, the first author's name, year of publication, study type, disease type, the number of patients, trial phase, treatment and control arms, the number of patients with intraoperative outcomes, postoperative outcomes and postoperative complications were extracted. The quality of the methodology used in randomized controlled trials (RCTs) was determined by the Jadad criteria (15). The quality of each trial was scored and grouped as either high- or low-quality trial. High-quality trials had scores of more than three (score $\geq 3$ ) while the low-quality trials had scores of less than two (score $\leq 2$ ). The Newcastle-Ottawa Scale (NOS) criteria (http://www.ohri. ca/programs/clinical_epidemiology/oxford.asp) was used to determine the quality of the methodology used in nonrandomized trials. Score ranged from 0 to 9 stars. High- 


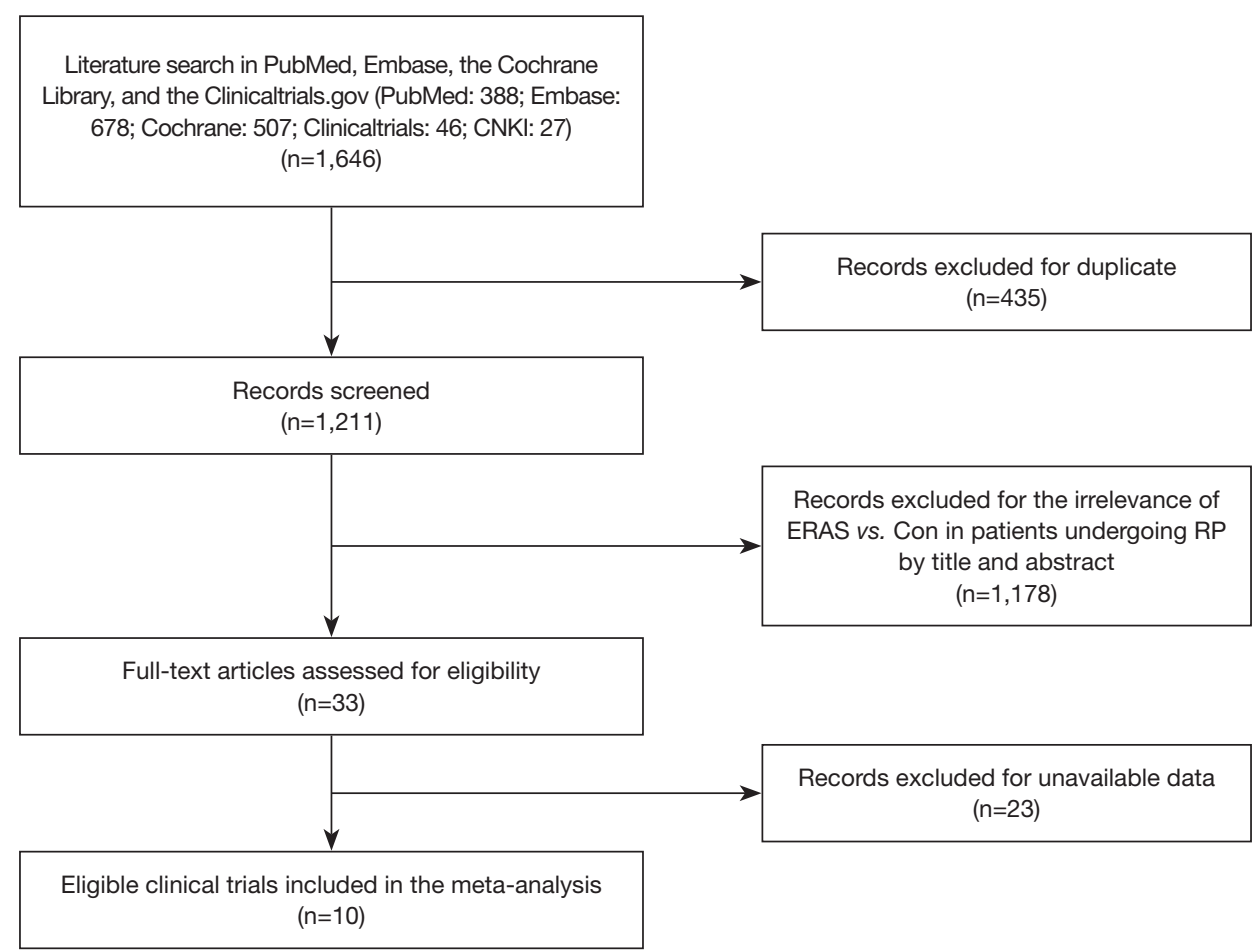

Figure 1 Flow chart for eligible studies. ERAS, enhanced recovery after surgery; Con, conventional group; RP, radical prostatectomy.

quality trials were those with scores of more than seven stars (score $\geq 7$ stars).

\section{Statistical analysis}

Data of patients with intraoperative outcomes, postoperative outcomes and postoperative complications was extracted from all the included trials. SMD, RR and 95\% CI were calculated to determine the association strength of these two regimens with the outcomes. The $\mathrm{Q}$ and $\mathrm{I}^{2}$ statistics were used to determine the heterogeneity. $\mathrm{I}^{2}$ of more than fifty percent $\left(\mathrm{I}^{2}>50 \%\right)$ indicated a statistically significant heterogeneity. The random-effect model was used in meta-analyses of the conservative statistics. Subgroup analysis was carried out based on the clinical characteristics. A funnel plot was used to determine any bias in the publications. Begg adjusted rank correlation test (16) and Egger regression test (17) were also performed to assess the publication bias. A sensitivity analysis was performed to determine whether the results of the meta-analysis were robust. STATA statistical version 12.0 software was used to perform all the statistical analyses (Stata Corporation, College Station, Texas, USA). A $P$ value of less than $0.05(\mathrm{P}<0.05)$ was considered statistically significant. All the $\mathrm{P}$ vales were two-sided.

\section{Results}

\section{Characteristics of studies included in this study}

Our initial search yielded 1,646 potentially relevant clinical trials on ERAS or conventional care in patients who underwent RP. After reviewing and screening, 10 primary studies (18-27) met our inclusion criteria. Among the ten, five were RCTs studies, four were retrospective trials and one was a prospective cohort study. The studies had 3,803 subjects that were pooled for meta-analyses (Figure 1). The baseline characteristics of each trial are shown in Table 1 while the Care elements implemented in ERAS protocol for $\mathrm{RP}$ in each trial are shown in Table 2. All trials included were open label and had between 50 and 2,610 patients enrolled for the trial. The Jadad quality scores of the included RCTs ranged from 2 to 3 while the NOS quality scores for the prospective cohort study and the retrospective trials ranged from 7 to 8 stars. Base on the eligibility criteria of most of the trials, patients with impaired hepatic, renal or bone marrow function were excluded. A majority of the patients in these trials had an Eastern Cooperative Oncology Group (ECOG) performance-status scores of 0 or 1 . This systematic review followed the guidelines of the PRISMA statement. 


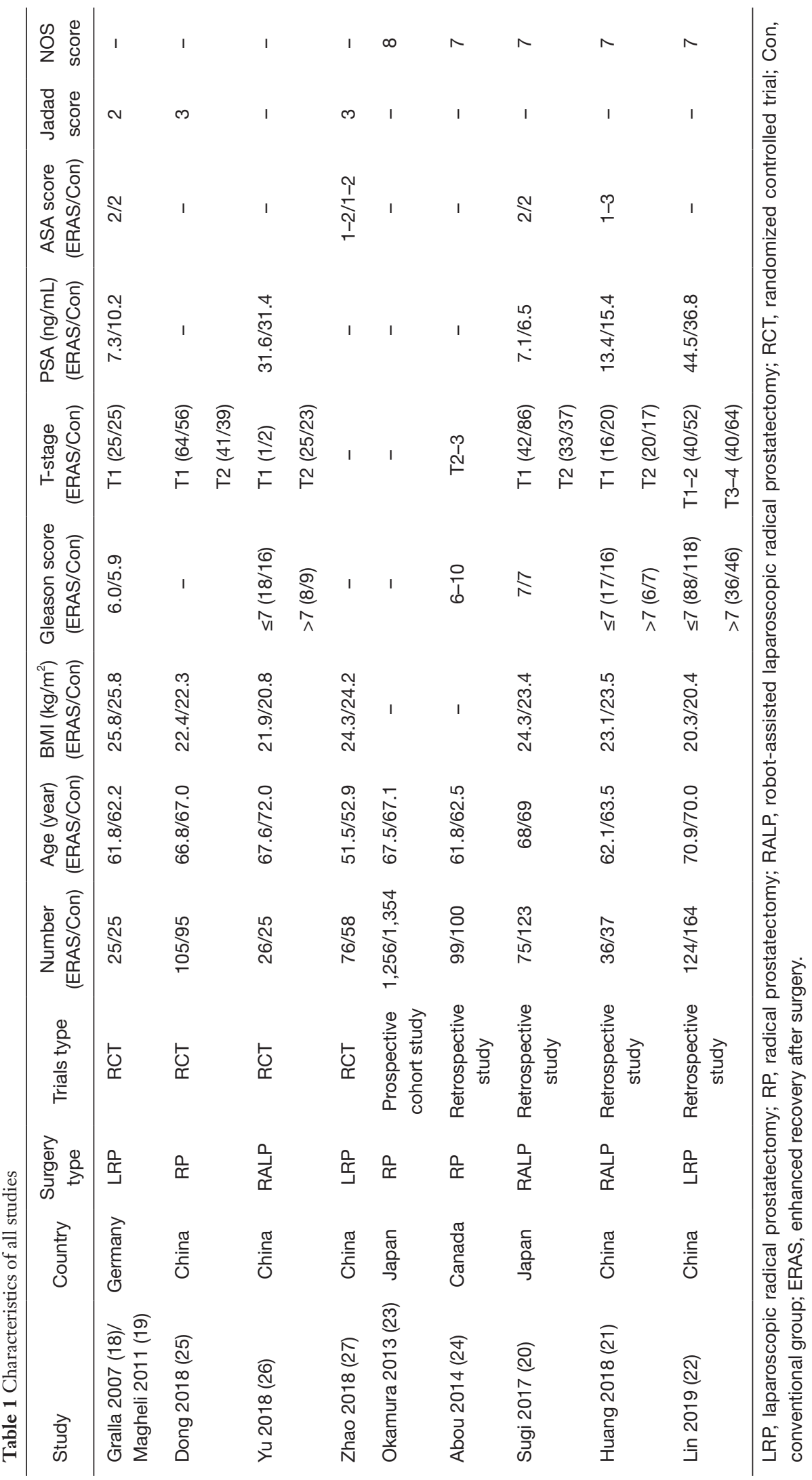


Table 2 Care elements implemented in ERAS protocol for RP

\begin{tabular}{|c|c|c|c|c|c|c|c|c|c|}
\hline ERAS elements & \multicolumn{9}{|c|}{ Eligible trials } \\
\hline \multicolumn{10}{|l|}{ Preoperative interventions } \\
\hline $\begin{array}{l}\text { Patient education/ } \\
\text { counseling }\end{array}$ & $\sqrt{ }$ & $\sqrt{ }$ & $\sqrt{ }$ & $\sqrt{ }$ & & $\sqrt{ }$ & & $\sqrt{ }$ & $\sqrt{ }$ \\
\hline Bowel preparation & $\sqrt{ }$ & & $\sqrt{ }$ & & & & $\sqrt{ }$ & $\sqrt{ }$ & \\
\hline Optimized diets & $\sqrt{ }$ & & & $\sqrt{ }$ & & $\sqrt{ }$ & $\sqrt{ }$ & & \\
\hline $\begin{array}{l}\text { Prophylactic } \\
\text { antibiotics }\end{array}$ & & & & & $\sqrt{ }$ & & & & $\sqrt{ }$ \\
\hline $\begin{array}{l}\text { Prophylactic } \\
\text { antiemesis }\end{array}$ & & & & & & & & & $\sqrt{ }$ \\
\hline \multicolumn{10}{|l|}{ Intraoperative interventions } \\
\hline $\begin{array}{l}\text { Prophylactic } \\
\text { antibiotics }\end{array}$ & $\sqrt{ }$ & & & & $\sqrt{ }$ & & & & \\
\hline Pneumoperitoneum & $\sqrt{ }$ & & & & & & & & \\
\hline Scrotal jockstrap & $\sqrt{ }$ & & & & & & & & \\
\hline $\begin{array}{l}\text { Epidural anesthesia/ } \\
\text { nonsteroidal analgesic } \\
\text { painkillers }\end{array}$ & $\sqrt{ }$ & & $\sqrt{ }$ & & & & & & $\sqrt{ }$ \\
\hline Use of drain-age tubes & & & & & $\sqrt{ }$ & $\sqrt{ }$ & & & $\sqrt{ }$ \\
\hline \multicolumn{10}{|l|}{ Postoperative interventions } \\
\hline $\begin{array}{l}\text { Nonsteroidal analgesic } \\
\text { painkillers }\end{array}$ & $\sqrt{ }$ & & & & & & $\sqrt{ }$ & $\sqrt{ }$ & \\
\hline $\begin{array}{l}\text { Intravenous fluid } \\
\text { restriction }\end{array}$ & $\sqrt{ }$ & & & & & & & $\sqrt{ }$ & $\sqrt{ }$ \\
\hline Early oral feeding & $\sqrt{ }$ & $\sqrt{ }$ & $\sqrt{ }$ & $\sqrt{ }$ & $\sqrt{ }$ & $\sqrt{ }$ & & $\sqrt{ }$ & $\sqrt{ }$ \\
\hline Early ambulation & $\sqrt{ }$ & $\sqrt{ }$ & $\sqrt{ }$ & $\sqrt{ }$ & $\sqrt{ }$ & $\sqrt{ }$ & $\sqrt{ }$ & $\sqrt{ }$ & $\sqrt{ }$ \\
\hline $\begin{array}{l}\text { Prophylactic } \\
\text { medication }\end{array}$ & & & & & $\sqrt{ }$ & & & & $\sqrt{ }$ \\
\hline $\begin{array}{l}\text { Early drainage tube } \\
\text { removal }\end{array}$ & & & $\sqrt{ }$ & $\sqrt{ }$ & $\sqrt{ }$ & $\sqrt{ }$ & & & $\sqrt{ }$ \\
\hline
\end{tabular}

ERAS, enhanced recovery after surgery; RP, radical prostatectomy. 
Table 3 Data of intraoperative outcomes

\begin{tabular}{|c|c|c|c|c|}
\hline Study & \multicolumn{2}{|c|}{ Operation time $(\min \pm \mathrm{SD})$} & \multicolumn{2}{|c|}{ Blood loss (mL \pm SD) } \\
\hline Gralla 2007/Magheli 2011 & $240 \pm 64.7$ & $220.1 \pm 57.0$ & $275.2 \pm 315.2$ & $156.9 \pm 71.8$ \\
\hline Okamura 2013 & $227 \pm 74$ & $215 \pm 67$ & $1,226 \pm 974$ & $1,209 \pm 880$ \\
\hline Sugi 2017 & $219 \pm 63.0$ & $225 \pm 67.3$ & $200 \pm 207.9$ & $200 \pm 349.5$ \\
\hline Lin 2019 & $102 \pm 24$ & $106 \pm 32.1$ & $151.1 \pm 32.5$ & $164.3 \pm 41.5$ \\
\hline Yu 2018 & $283 \pm 57.7$ & $273.5 \pm 70.4$ & $197.2 \pm 165.2$ & $187.9 \pm 125.2$ \\
\hline
\end{tabular}

ERAS, enhanced recovery after surgery; Con, conventional group; SD, standard deviation.

Table 4 Data of postoperative outcomes

\begin{tabular}{|c|c|c|c|c|c|c|c|c|c|c|}
\hline \multirow[t]{2}{*}{ Study } & \multicolumn{2}{|c|}{$\begin{array}{l}\text { Hospital stay } \\
\text { (days } \pm \text { SD) }\end{array}$} & \multicolumn{2}{|c|}{$\begin{array}{l}\text { Catheter stay } \\
\text { (days } \pm \text { SD) }\end{array}$} & \multicolumn{2}{|c|}{$\begin{array}{l}\text { First defecation } \\
\quad \text { (days } \pm \mathrm{SD})\end{array}$} & \multicolumn{2}{|c|}{ Pain (n) } & \multicolumn{2}{|c|}{$\begin{array}{l}\text { First anal exhaust } \\
\text { (days } \pm \text { SD) }\end{array}$} \\
\hline & ERAS & Con & ERAS & Con & ERAS & Con & ERAS & Con & ERAS & Con \\
\hline $\begin{array}{l}\text { Gralla 2007/ } \\
\text { Magheli } 2011\end{array}$ & $3.6 \pm 1.2$ & $6.7 \pm 0.9$ & $11.4 \pm 10.7$ & $9.6 \pm 6.9$ & $3.6 \pm 1.2$ & $6.7 \pm 0.9$ & 4 & 15 & - & - \\
\hline Okamura 2013 & $13.6 \pm 6.1$ & $15.3 \pm 7.1$ & $8.3 \pm 7.1$ & $8.7 \pm 5.3$ & - & - & - & - & - & - \\
\hline Sugi 2017 & $10.5 \pm 3.3$ & $9 \pm 6.9$ & $6 \pm 2.3$ & $6 \pm 7.4$ & $2.6 \pm 1.0$ & $3 \pm 1.3$ & - & - & - & - \\
\hline Huang 2018 & $7.3 \pm 1.6$ & $9.8 \pm 2.0$ & - & - & - & - & - & - & $2.6 \pm 1.1$ & $3.6 \pm 1.5$ \\
\hline Lin 2019 & $3.8 \pm 1.7$ & $9.2 \pm 2.7$ & $6.5 \pm 0.5$ & $6.6 \pm 0.7$ & $0.7 \pm 0.2$ & $3.4 \pm 1.5$ & - & - & $0.4 \pm 0.3$ & $1.3 \pm 1.0$ \\
\hline
\end{tabular}

$\mathrm{SD}$, standard deviation.

\section{Findings-intraoperative outcomes (operation time and blood loss)}

A total of 3,270 subjects treated with either ERAS or conventional care in six trials were included for the analysis of operation time and blood loss (data shown in Tables 3,4). An SMD of 0.00 (95\% CI: $-0.19,0.20, \mathrm{I}^{2}=63.4 \%$ ) were obtained from analysis of the operation times of patients under ERAS and conventional care. In the analysis of blood loss, an SMD of -0.00 (95\% CI: $-0.19,0.19, \mathrm{I}^{2}=60.2 \%$ ) were obtained (Figure 2). These results showed no statistical difference in both the operation time and blood loss between ERAS and conventional care groups $(\mathrm{P}=0.987)$.

Although the results of intraoperative outcomes indicated statistically significant heterogeneity, the sensitivity analysis showed that the results of intraoperative outcomes were robust (Figure S1).

\section{Findings-postoperative outcomes (bospital stay, catbeter} stay, first defecation and first anal exhaust)

\section{Hospital stay}

A total of 3,803 subjects treated with either ERAS or conventional care in nine trials were included for the analysis of hospital stay (data shown in Tables 3,4). An SMD of -1.65 (95\% CI: $-2.53,-0.76, \mathrm{I}^{2}=98.7 \%$ ) was obtained from analysis of hospital stay of patients under ERAS and conventional care (Figure 3). The results showed that ERAS group had a shorter hospital stay than conventional 


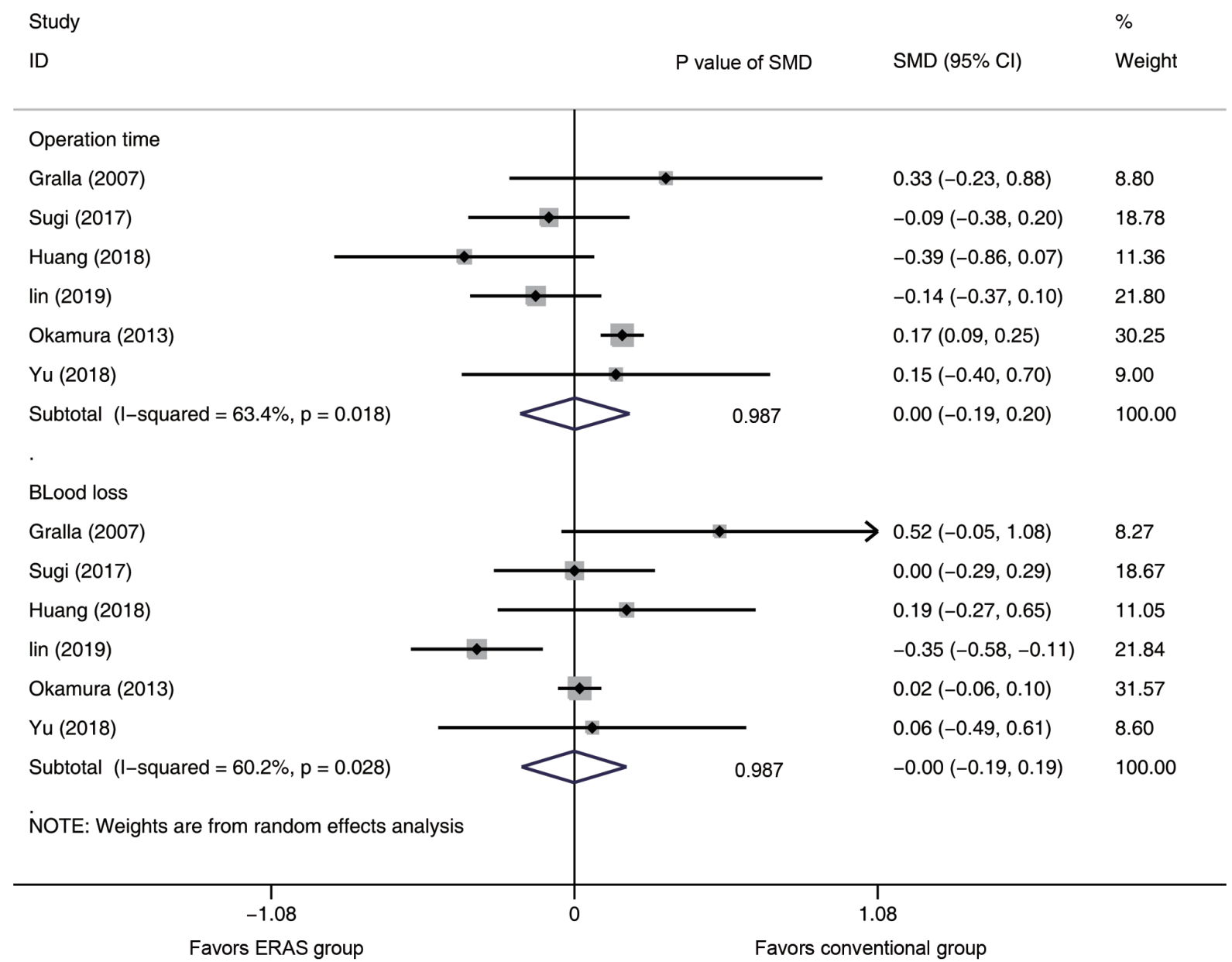

Figure 2 Annotated forest plot for meta-analysis of intraoperative outcomes of ERAS and conventional groups. Summary of intraoperative outcomes (operation time and blood loss) SMD between ERAS and conventional groups were calculated using the random effect model. Size of squares is directly proportional to the amount of information available. ERAS, enhanced recovery after surgery; SMD, standard mean difference; CI, confidence interval; P, P value of the Q test for heterogeneity.

care groups $(\mathrm{P}<0.001)$. As the results indicated statistically significant heterogeneity, subgroup analysis was performed to find sources of heterogeneity. The nine trials were first separated in three groups (LRP, RALP and RP) based on their surgery sub-type. However, the results still showed heterogeneity (Table 5) indicating that the difference in surgery sub-types was not the source of heterogeneity. However, the sensitivity analysis showed that the results of hospital stay were robust (Figure S2).

\section{Catheter stay}

A total of 3,197 subjects treated with either ERAS or conventional care in five trials were included for the analysis of catheter stay (data shown in Tables 3,4). In the analysis of catheter stay, an SMD of -0.12 (95\% CI: $-0.31,0.07$, $\mathrm{I}^{2}=59.8 \%$ ) were obtained (Figure 3). The results showed no statistical difference in catheter stay between ERAS and conventional care groups $(\mathrm{P}=0.204)$.

\section{First defecation}

Six hundred and seventy subjects treated with either ERAS or conventional care in four trials were included for the analysis of first defecation (data shown in Tables 3,4). An SMD of -1.56 (95\% CI: $\left.-2.71,-0.42, \mathrm{I}^{2}=97.4 \%\right)$ were obtained (Figure 3). The results showed that ERAS groups had a shorter time to first defecation compared to the conventional care groups $(\mathrm{P}=0.008)$. The results indicated statistically significant heterogeneity. Subgroup analysis was 


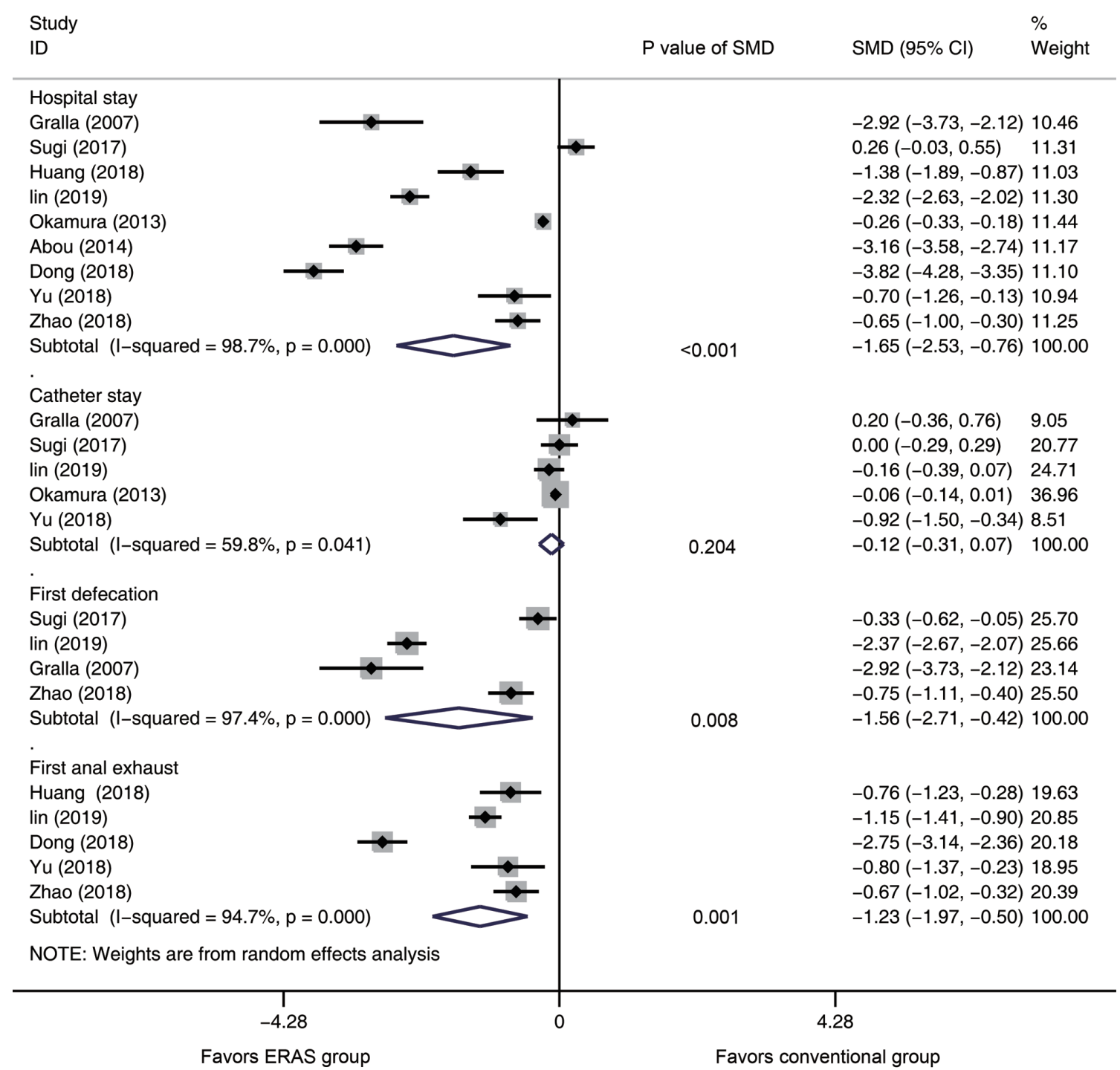

Figure 3 Annotated forest plot for meta-analysis of postoperative outcomes of ERAS and conventional groups. Summary of postoperative outcomes (hospital stay, catheter stay, first defecation and first anal exhaust) SMD between ERAS and conventional groups were calculated using the random effect model. Size of squares is directly proportional to the amount of information available. ERAS, enhanced recovery after surgery; SMD, standard mean difference; P, P value of the Q test for heterogeneity.

performed to find sources of heterogeneity. The four trials were first separated in two groups (LRP and RALP) based on their surgery sub-type. However, the results still showed heterogeneity (Table 5) indicating that the difference in surgery sub-types was not the source of heterogeneity. But sensitivity analysis showed that the results of first defecation were robust (Figure S3).

\section{First anal exhaust}

Seven hundred and forty-eight subjects treated with either
ERAS or conventional care in 5 trials were included for the analysis of first anal exhaust (data shown in Tables 3,4). An SMD of -1.23 (95\% CI: $\left.-1.97,-0.50, \mathrm{I}^{2}=94.7 \%\right)$ were obtained (Figure 3). The results showed that ERAS group had a shorter time to first anal exhaust than conventional care groups $(\mathrm{P}=0.001)$. The results indicated statistically significant heterogeneity. Subgroup analysis was performed to find sources of heterogeneity. The five trials were first separated in three groups (LRP, RALP and RP) based on their surgery sub-type. However, the results still showed 
Table 5 Subgroup analysis of operation time, first defecation and first anal exhaust

\begin{tabular}{|c|c|c|c|c|c|c|}
\hline Subgroup analysis & $\mathrm{N}$ & $\mathrm{RR}(95 \% \mathrm{Cl})$ & $P$ values & \multicolumn{3}{|c|}{ Test for heterogeneity } \\
\hline LRP & 3 & $-1.98(-3.26,-0.71)$ & 0.002 & 54.64 & $<0.001$ & $96.3 \%$ \\
\hline RALP & 1 & $-0.34(-0.62,-0.05)$ & 0.023 & - & - & - \\
\hline LRP & 2 & $-0.93(-1.40,-0.46)$ & $<0.001$ & 4.76 & 0.029 & $79.0 \%$ \\
\hline RALP & 2 & $-0.78(-1.14,-0.41)$ & $<0.001$ & 0.01 & 0.913 & $0 \%$ \\
\hline $\mathrm{RP}$ & 1 & $-2.75(-3.14,-2.36)$ & $<0.001$ & - & - & - \\
\hline \multicolumn{7}{|l|}{ Hospital stay } \\
\hline $\mathrm{RP}$ & 3 & $-2.40(-4.96,0.15)$ & 0.065 & 385.13 & $<0.001$ & $99.5 \%$ \\
\hline
\end{tabular}

LRP, laparoscopic radical prostatectomy; RP, radical prostatectomy; RALP, robot-assisted laparoscopic radical prostatectomy; N, number of trials; $R R$, risk ratio; $\mathrm{Cl}$, confidence interval; $P_{h}, P$ value of the $Q$ test for heterogeneity.

heterogeneity (Table 5) indicating that the difference in surgery sub-types was not the source of heterogeneity. But sensitivity analysis showed that the results of first anal exhaust were robust (Figure S4).

\section{Findings—postoperative complications}

Several different adverse events and toxicities were reported (data shown in Table 6). In the meta-analysis, patients treated with either ERAS or conventional care from ten studies were included for analysis of postoperative complications. ERAS group had significant lower incidence of nausea than the conventional care group (overall RR $=0.62,95 \%$ CI: $0.40,0.94, P=0.024)$. However, there was no statistical difference of other postoperative complications listed in Table 7 between ERAS and conventional care arms $(\mathrm{P}>0.05)$.

\section{Publication bias}

The shape of the funnel plot did not display any evidence of apparent asymmetry. Furthermore, the formal tests showed no substantial publication bias. The Egger's test had a $\mathrm{P}$ value of 0.463 while the Begg's test had a $\mathrm{P}$ value of 0.115 (Figure S5).

\section{Discussion}

In recent years, minimally invasive $\mathrm{RP}$ and open $\mathrm{RP}$ have been widely used for the management of Pca patients. Minimally invasive RP is advantageous because it minimizes operative injuries and reduces complications compared to open surgery. However, there seemed to be no significant difference in efficacy between these two sub-types of RP $(4,28,29)$. In some countries, open RP is still an option for Pca patients. Currently, many medical centers have implemented the ERAS into RP programs. The ERAS protocol is a standardized perioperative care pathway aimed at minimizing the stress of surgery, reduce postoperative morbidity, shorten hospital stay and accelerate recovery. ERAS has been widely implemented in abdominal and gynecologic surgery $(7,30,31)$. Nonetheless, results from clinical trials are not compelling enough to support any definitive conclusions about the superiority of ERAS in RP programs. In this study, we performed a systematic review and meta-analysis on the efficacy and safety of ERAS and conventional care for Pca patients undergoing RP from 10 primary studies. Among the ten, five were RCTs, four were retrospective trials and one was a prospective cohort study. Results indicated that the ERAS group had significantly shorter hospital stay, shorter time to first defecation, shorter time to first anal exhaust, and lower incidence of nausea 


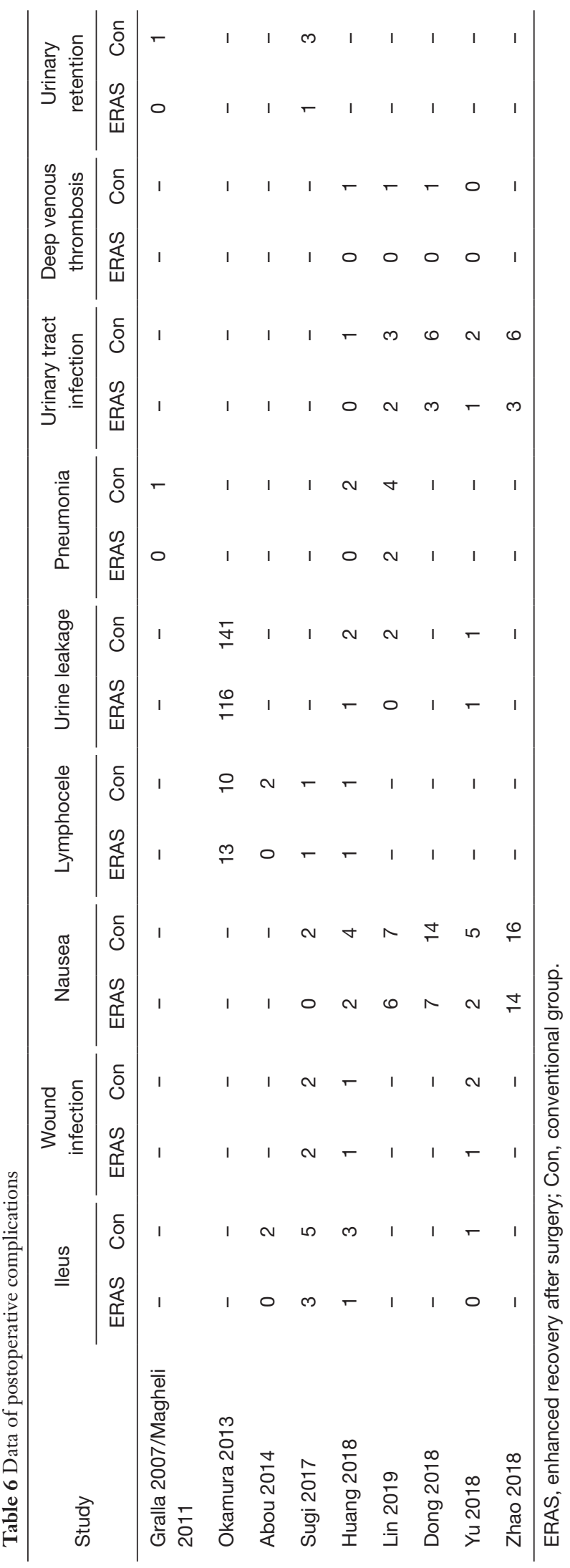

compared to the conventional group. However, there was no statistical difference in intraoperative outcomes, catheter stay and other postoperative complications between ERAS and conventional group.

Overall survival (OS) and recurrence-free survival (RFS) are the key outcomes for evaluating the efficacy of a surgery. However, the trials included in this meta-analysis did not assess the OS nor RFS, because they were all shortterm studies. As such, long-term clinical trials are needed to compare the OS and RFS of these two groups. ERAS group had significantly shorter hospital stay compared to the conventional care group $(\mathrm{P}<0.001)$. However, the difference in intraoperative outcomes and catheter stay between ERAS and conventional care arms was not significant $(\mathrm{P}>0.05)$. Hospital stay is an important outcome for any surgery. Sugi 2017 reported that there was no significant difference in hospital stay between these two groups $(\mathrm{P}>0.05)$ (data shown in Tables 3,4) (20). Contrary to these findings, the ERAS group had a significantly shorter hospital stay period compared to the conventional group $(\mathrm{P}<0.05)$ in 8 trials used in this study (19,21-27). Similarly, Lin 2018 compared the hospitalization costs between these two groups and found out that the ERAS group could significantly reduce the hospitalization costs compared to the conventional care group (6.1 vs. 7.2 thousand USD, $\mathrm{P}<0.001)$ (22).

Meta-analysis results also revealed that the ERAS group had significantly shorter times to first defecation $(\mathrm{P}=0.008)$ and first anal exhaust compared to the conventional group $(\mathrm{P}=0.001)$. Time to first defecation and anal exhaust were two indicators of postoperative recovery of intestinal function. The ERAS protocol suggested omission of preoperative bowel preparation, preoperative carbohydrate loading, and restricted fluid therapy. Evidently, ERAS group had a better postoperative intestinal function recovery than the conventional group.

Another potential advantage of ERAS would be the reduction of the frequencies of postoperative complications. Huang 2018 reported that ERAS could significantly reduce the incidence of pain compared to the conventional group (data shown in Tables 3,4, $\mathrm{P}=0.004$ ) (21). In the same line, Okamura 2013 reported that there was significantly lower incidence of fever above $38{ }^{\circ} \mathrm{C}$ in ERAS group compared to the conventional group (1.9\% vs. $3.5 \%, \mathrm{P}=0.014)$ (23). Similarly, Gralla 2007 reported that there was significantly lower incidence of penoscrotal complications in the ERAS group compared to the conventional group ( 5 vs. 12 , $\mathrm{P}=0.04)$ (18). However, trials included for this study were not enough to accurately assess these complications between 
Table 7 Meta-analysis results of the incidences of postoperative complications between ERAS and conventional groups in patients undergoing RP

\begin{tabular}{|c|c|c|c|c|c|c|}
\hline Postoperative complications & $\mathrm{N}$ & $\mathrm{RR}(95 \% \mathrm{Cl})$ & $P$ values & \multicolumn{3}{|c|}{ Test for heterogeneity } \\
\hline Ileus & 4 & $0.57(0.20,1.62)$ & 0.293 & 1.36 & 0.714 & $0 \%$ \\
\hline Wound infection & 3 & $1.00(0.27,3.71)$ & 0.998 & 0.63 & 0.731 & $0 \%$ \\
\hline Nausea* $^{*}$ & 6 & $0.62(0.40,0.94)$ & 0.024 & 2.39 & 0.793 & $0 \%$ \\
\hline Urine leakage & 4 & $0.88(0.70,1.10)$ & 0.263 & 0.82 & 0.846 & $0 \%$ \\
\hline Pneumonia & 3 & $0.46(0.12,1.76)$ & 0.260 & 0.50 & 0.780 & $0 \%$ \\
\hline Urinary tract infection & 5 & $0.48(0.22,1.04)$ & 0.061 & 0.61 & 0.961 & $0 \%$ \\
\hline Deep venous thrombosis & 3 & $0.36(0.06,2.24)$ & 0.272 & 0.03 & 0.986 & $0 \%$ \\
\hline
\end{tabular}

*, there had statistically difference between two arms. ERAS, enhanced recovery after surgery; RP, radical prostatectomy; Cl, confidence interval; $N$, number of trials; RR, risk ratio; $P_{h}, P$ value of the $Q$ test for heterogeneity.

the two groups. As such, no definite conclusion could be drawn. Nonetheless, pooled meta-analysis results revealed that the ERAS group had significant lower incidence of nausea compared to the conventional care group. However, there were no significant differences in other postoperative complications between ERAS and conventional groups $(\mathrm{P}>0.05)$ (data shown in Table 7).

Heterogeneity is an important aspect in meta-analysis. In this study, statistical analysis revealed that heterogeneity was present in most aspects. Subgroup analysis performed indicated that the difference in surgery sub-types was not the source of heterogeneity. Although the sources of heterogeneity were not found, sensitivity analysis results indicated that the overall significance of the pooled estimates were not affected by any trial included in the study. Similarly, Begg's and Egger's tests were used to detect any publication bias that would introduce false positives in meta-analysis (17). No evidence of publication bias was detected. These results indicated that all the conclusions of this study were credible and verifiable.

However, this study is limited by several factors. The number of studies used was low because of lack of enough high-quality RCTs. As such, some complications could not be accurately assessed. Further to this, the trials included in this study were short-term studies, had inconsistent ERAS protocols and were open labelled. All these were factors that could have affected the outcomes of the study. In future studies, more rigorous long-term experiments needed to be designed to enable precise meta-analysis of all aspects between the ERAS and conventional care groups.

\section{Conclusions}

Evidently, the ERAS group had significantly shorter hospital stay, shorter time to first defecation, shorter time to first anal exhaust and lower incidence of nausea compared to the conventional care group. Both groups had similar incidences of other postoperative complications. Based on the consistence of the data presented so far in this and previous studies, ERAS has the potential to be used as a standard of care for Pca patients undergoing RP.

\section{Acknowledgments}

We thank our collaborators who helped with this study.

Funding: This work was supported by Joint Fund of Zhejiang Provincial Natural Science Foundation, China (Grant number LYY18H310002), Funding of Zhejiang Pharmaceutical Association (Grant number ZYYZL01) and Medical Health Science and Technology Project of Zhejiang Provincial Health Commission, China (Grant number 2017KY345).

\section{Footnote}

Conflicts of Interest: All authors have completed the ICMJE 
uniform disclosure form (available at http://dx.doi. org/10.21037/apm.2020.04.03). The authors have no conflicts of interest to declare.

Ethical Statement: The authors are accountable for aspects of the work in ensuring that questions related to the accuracy or integrity of any part of the work are appropriately investigated and resolved.

Open Access Statement: This is an Open Access article distributed in accordance with the Creative Commons Attribution-NonCommercial-NoDerivs 4.0 International License (CC BY-NC-ND 4.0), which permits the noncommercial replication and distribution of the article with the strict proviso that no changes or edits are made and the original work is properly cited (including links to both the formal publication through the relevant DOI and the license). See: https://creativecommons.org/licenses/by-nc-nd/4.0/.

\section{References}

1. Siegel RL, Miller KD, Jemal A. Cancer Statistics, 2017. CA Cancer J Clin 2017;67:7-30.

2. Mottet N, Bellmunt J, Bolla M, et al. EAU-ESTROSIOG Guidelines on Prostate Cancer. Part 1: Screening, Diagnosis, and Local Treatment with Curative Intent. Eur Urol 2017;71:618-29.

3. Ilic D, Evans SM, Allan CA, et al. Laparoscopic and robotassisted vs open radical prostatectomy for the treatment of localized prostate cancer: a Cochrane systematic review. BJU Int 2018;121:845-53.

4. Allan C, Ilic D. Laparoscopic versus Robotic-Assisted Radical Prostatectomy for the Treatment of Localised Prostate Cancer: A Systematic Review. Urol Int 2016;96:373-8.

5. Ficarra V, Novara G, Artibani W, et al. Retropubic, laparoscopic, and robot-assisted radical prostatectomy: a systematic review and cumulative analysis of comparative studies. Eur Urol 2009;55:1037-63.

6. Bekelman JE, Rumble RB, Chen RC, et al. Clinically Localized Prostate Cancer: ASCO Clinical Practice Guideline Endorsement of an American Urological Association/American Society for Radiation Oncology/ Society of Urologic Oncology Guideline. J Clin Oncol 2018;36:3251-8.

7. Kehlet H. Multimodal approach to control postoperative pathophysiology and rehabilitation. Br J Anaesth 1997;78:606-17.
8. Li S, Zhou K, Che G, et al. Enhanced recovery programs in lung cancer surgery: systematic review and metaanalysis of randomized controlled trials. Cancer Manag Res 2017;9:657-70.

9. Pedziwiatr M, Mavrikis J, Witowski J, et al. Current status of enhanced recovery after surgery (ERAS) protocol in gastrointestinal surgery. Med Oncol 2018;35:95.

10. Lemanu DP, Singh PP, Stowers MD, et al. A systematic review to assess cost effectiveness of enhanced recovery after surgery programmes in colorectal surgery. Colorectal Dis 2014;16:338-46.

11. Li Z, Wang Q, Li B, et al. Influence of enhanced recovery after surgery programs on laparoscopy-assisted gastrectomy for gastric cancer: a systematic review and meta-analysis of randomized control trials. World J Surg Oncol 2017;15:207.

12. Offodile AC 2nd, Gu C, Boukovalas S, et al. Enhanced recovery after surgery (ERAS) pathways in breast reconstruction: systematic review and meta-analysis of the literature. Breast Cancer Res Treat 2019;173:65-77.

13. Azhar RA, Bochner B, Catto J, et al. Enhanced Recovery after Urological Surgery: A Contemporary Systematic Review of Outcomes, Key Elements, and Research Needs. Eur Urol 2016;70:176-87.

14. Moher D, Liberati A, Tetzlaff J, et al. Preferred reporting items for systematic reviews and meta-analyses: the PRISMA statement. BMJ 2009;339:b2535.

15. Jadad AR, Moore RA, Carroll D, et al. Assessing the quality of reports of randomized clinical trials: is blinding necessary? Control Clin Trials 1996;17:1-12.

16. Begg CB, Mazumdar M. Operating characteristics of a rank correlation test for publication bias. Biometrics 1994;50:1088-101.

17. Egger M, Davey Smith G, Schneider M, et al. Bias in meta-analysis detected by a simple, graphical test. BMJ 1997;315:629-34.

18. Gralla O, Haas F, Knoll N, et al. Fast-track surgery in laparoscopic radical prostatectomy: basic principles. World J Urol 2007;25:185-91.

19. Magheli A, Knoll N, Lein M, et al. Impact of fast-track postoperative care on intestinal function, pain, and length of hospital stay after laparoscopic radical prostatectomy. J Endourol 2011;25:1143-7.

20. Sugi M, Matsuda T, Yoshida T, et al. Introduction of an Enhanced Recovery after Surgery Protocol for RobotAssisted Laparoscopic Radical Prostatectomy. Urol Int 2017;99:194-200.

21. Huang Z, Yi L, Zhong Z, et al. Comparison of Fast- 
Track Versus Conventional Surgery Protocol for Patients Undergoing Robot-Assisted Laparoscopic Radical Prostatectomy: A Chinese Experience. Sci Rep 2018;8:8017.

22. Lin C, Wan F, Lu Y, et al. Enhanced recovery after surgery protocol for prostate cancer patients undergoing laparoscopic radical prostatectomy. J Int Med Res 2019;47:114-21.

23. Okamura K, Nojiri Y, Tanaka Y, et al. Changes in perioperative management of radical prostatectomy using clinical pathways according to a standardized care plan: a multi-institutional study. Int J Urol 2013;20:337-43.

24. Abou-Haidar H, Abourbih S, Braganza D, et al. Enhanced recovery pathway for radical prostatectomy: Implementation and evaluation in a universal healthcare system. Can Urol Assoc J 2014;8:418-23.

25. Dong N, Chan Y, Jia L, et al. Effect of rapid rehabilitation concept on postoperative treatment effect, compliance of pelvic floor muscle rehabilitation and urinary function of patients with prostate cancer. Oncol Progress 2018;16:1933-36.

26. Yu H, Wang J. ERAS in Multidisciplinary Cooperation in

Cite this article as: Ye Z, Chen J, Shen T, Yang H, Qin J, Zheng F, Rao Y. Enhanced recovery after surgery (ERAS) might be a standard care in radical prostatectomy: a systematic review and meta-analysis. Ann Palliat Med 2020;9(3):746-758. doi: 10.21037/apm.2020.04.03
Patients with Robot-assisted Laparoscopic. J Qilu Nursing 2018;24:18-21.

27. Zhao B, Shi L, Yuan Y, et al. Application of FTS conceptguided measures in perioperative care in laparoscopic radical prostatectomy. Chin J Clin Oncol Rehabil 2018;25:717-20.

28. Sujenthiran A, Nossiter J, Parry M, et al. National cohort study comparing severe medium-term urinary complications after robot-assisted vs laparoscopic vs retropubic open radical prostatectomy. BJU Int 2018;121:445-52.

29. Ilic D, Evans SM, Allan CA, et al. Laparoscopic and robotic-assisted versus open radical prostatectomy for the treatment of localised prostate cancer. Cochrane Database Syst Rev 2017;9:CD009625.

30. Li L, Chen J, Liu Z, et al. Enhanced recovery program versus traditional care after hepatectomy: A meta-analysis. Medicine (Baltimore) 2017;96:e8052.

31. Trowbridge ER, Dreisbach CN, Sarosiek BM, et al. Review of enhanced recovery programs in benign gynecologic surgery. Int Urogynecol J 2018;29:3-11. 


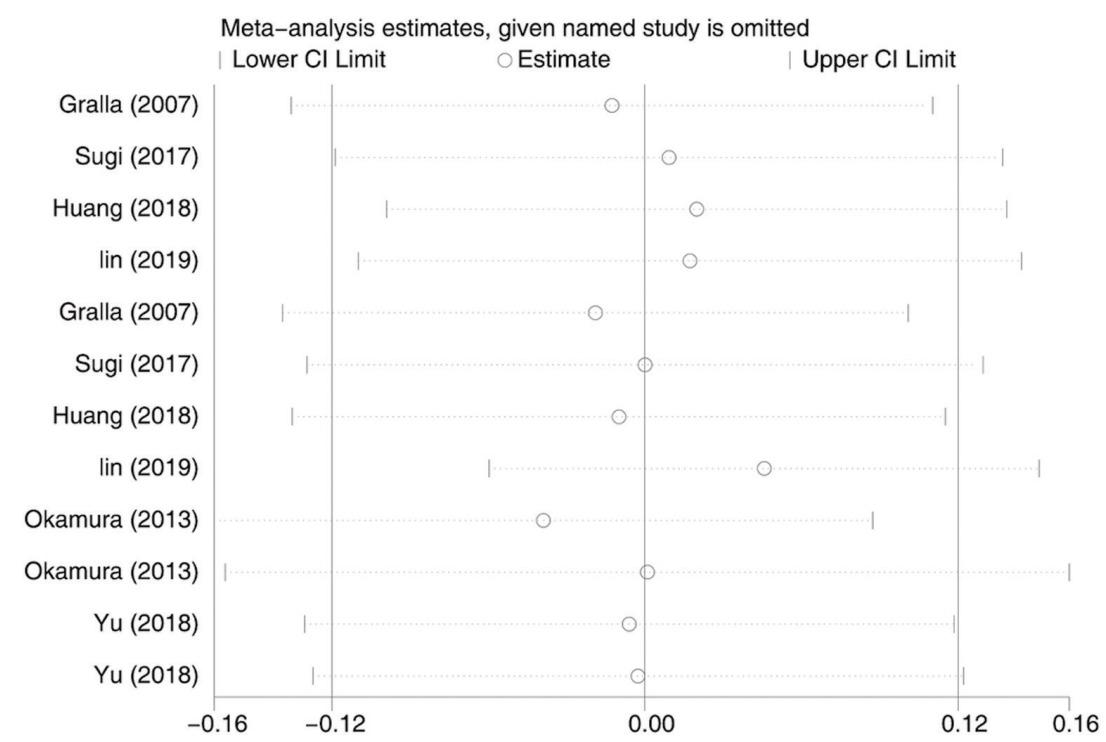

Figure S1 Sensitivity analysis of intraoperative outcomes.

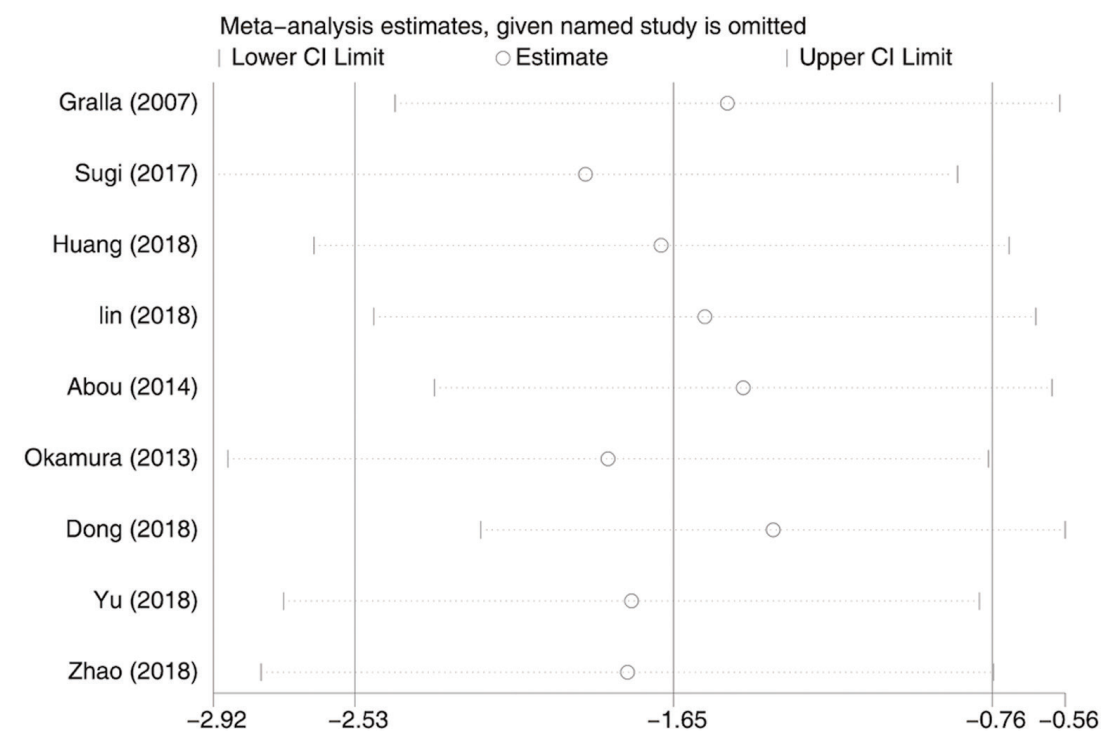

Figure S2 Sensitivity analysis of hospital stay outcome.

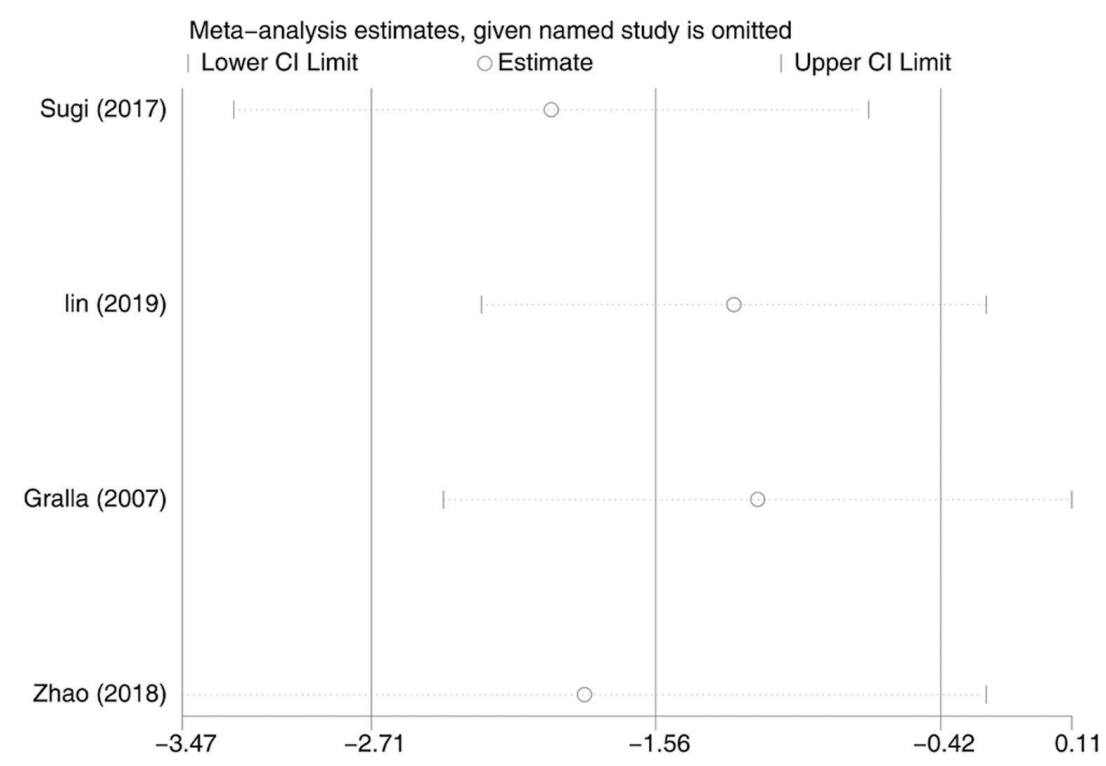

Figure S3 Sensitivity analysis of time to first defecation outcome. 


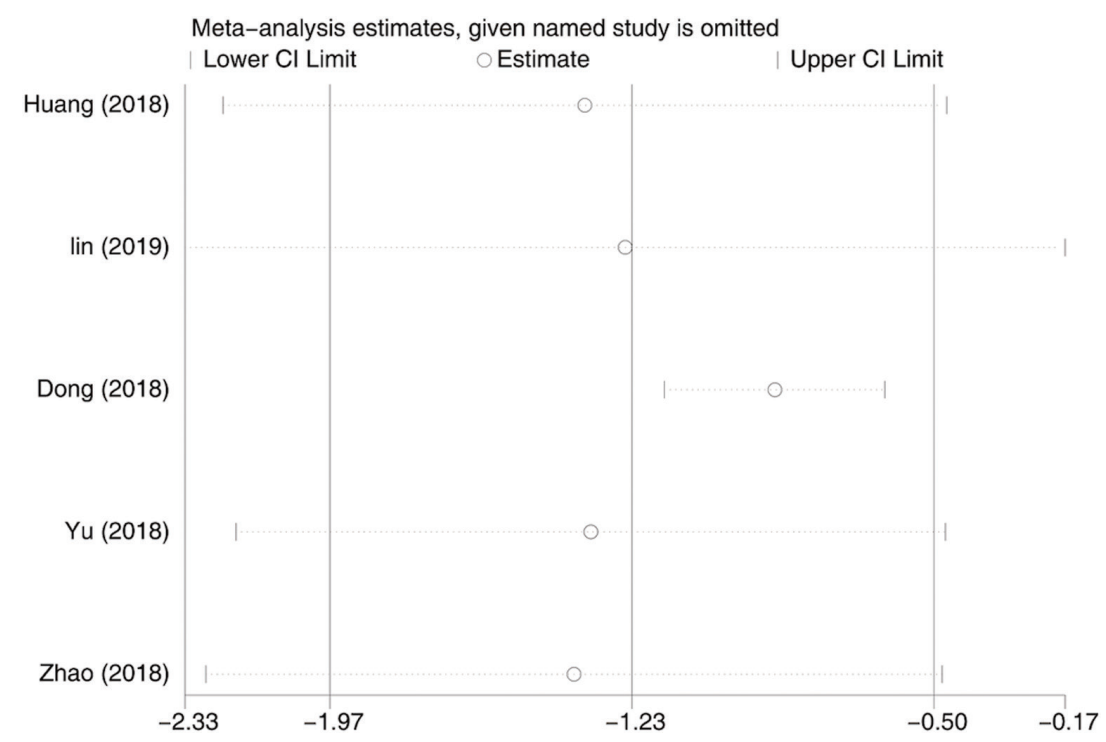

Figure S4 Sensitivity analysis of time to first anal exhaust outcome.

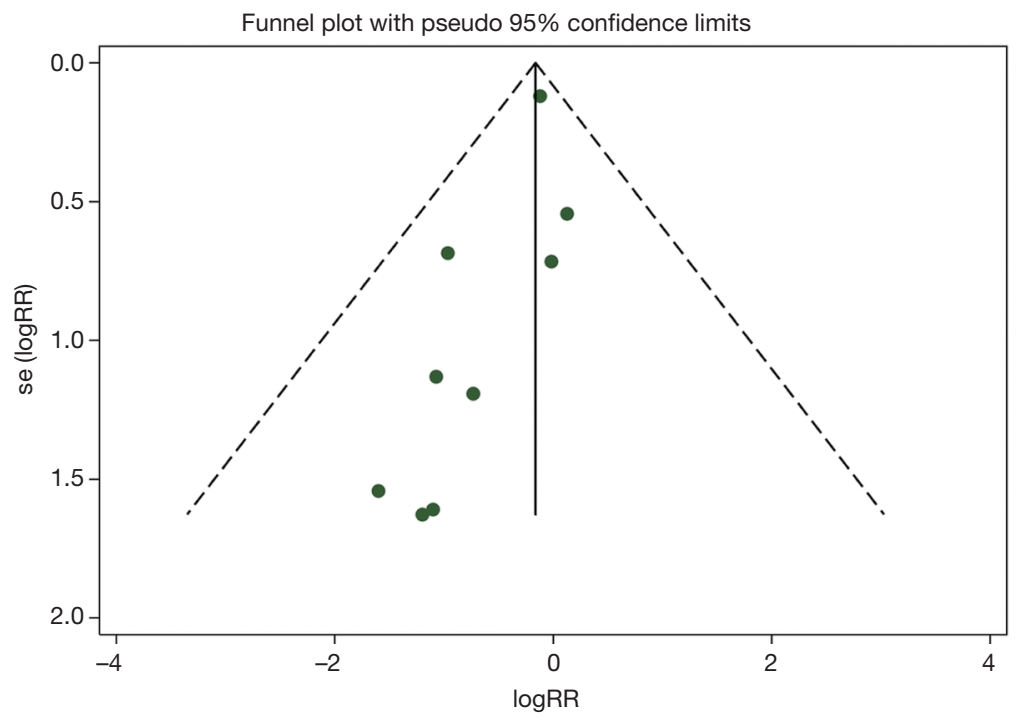

Figure S5 Publication bias risk. RR, risk ratio; se, standard error of the mean. 